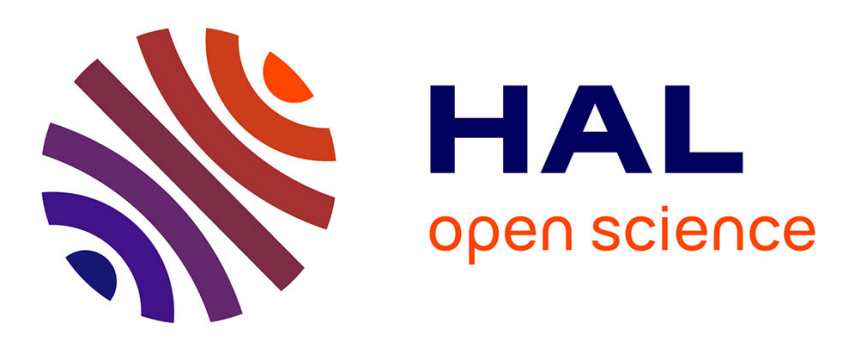

\title{
La représentation sémantique du mot inostranec dans les discours médiatiques russes
}

\author{
Vladimir Beliakov
}

\section{To cite this version:}

Vladimir Beliakov. La représentation sémantique du mot inostranec dans les discours médiatiques russes. La Revue russe, 2009, 33, pp.213-220. hal-00955271

\section{HAL Id: hal-00955271 \\ https://hal-univ-tlse2.archives-ouvertes.fr/hal-00955271}

Submitted on 4 Mar 2014

HAL is a multi-disciplinary open access archive for the deposit and dissemination of scientific research documents, whether they are published or not. The documents may come from teaching and research institutions in France or abroad, or from public or private research centers.
L'archive ouverte pluridisciplinaire HAL, est destinée au dépôt et à la diffusion de documents scientifiques de niveau recherche, publiés ou non, émanant des établissements d'enseignement et de recherche français ou étrangers, des laboratoires publics ou privés. 
Vladimir Beliakov

LA REPRESENTATION SEMANTIQUE DU MOT INOSTRANEC DANS LES DISCOURS MEDIATIQUES RUSSES

\section{Introduction}

Le but de cet article est de décrire la représentation sémantique du nom иностранеи relevée dans les discours médiatiques russes ${ }^{1}$. Je ne proposerai, ni développerai ici de thèses originales sur la représentation sémantique et la signification lexicale des mots. Je me contenterai, dans un premier temps, d'esquisser de façon succincte les principes fondamentaux de la théorie de la représentation sémantique, la théorie des stéréotypes, telle qu'elle a été conçue par H. Putnam et développée par B. Fradin et J-C. Anscombre et d'expliciter, dans un second temps, son fonctionnement sur l'exemple du mot иностранеи montrant ainsi l'apport que cette théorie est susceptible d'apporter aux études sémantiques et à l'analyse du discours.

Je vais m'appuyer sur un corpus constitué des articles de presse et des interviews publiés dans les journaux russes et en ligne sur les portails www.rambler.ru и шжw.yandex.ru entre 2001 et 2005, ainsi que sur des exemples tirés du Corpus national de la langue russe ${ }^{2}$.

\section{Définition lexicographique}

Dans les dictionnaires consultés, le mot иностранец est défini en dehors de tout contexte comme citoyen d'un autre pays : 
- гражданин, подданный какой-л. страны по отношению к другой стране. (Ефремова, 2000)

- лицо, принадлежащее к гражданам чужой страны. (Ушаков, 2000)

- гражданин какой-нибудь страны по отношению к другой стране. (Ожегов, 1981)

Ce trait descriptif du référent du nom implique que la phrase Иностранец имеет чужое гражданство soit affectée de la valeur de vérité car elle est conforme à la forme propositionnelle il est vrai que $P$. On peut donc considérer cette phrase en tant que phrase définitionnelle appartenant de façon nécessaire et suffisante à la représentation sémantique de иностранец car le fait d'être citoyen d'un autre pays est une propriété requise par définition pour que quelqu'un soit appelé иностранеи: s'il n'est pas vrai que $x$ имеет чужое гражданство on ne pourra pas le désigner par le terme иностранеu. J'adopte ici la notion de désignateur rigide qui selon $\mathrm{S}$. Kripke réfère dans une phrase donnée au même objet ou même individu dans tout monde possible où ce désignateur (substantif) désigne (Putnam, 1985 : 29). Autrement dit, un terme est rigide s'il porte un effet de référence au même individu ou objet dans n'importe quel discours : tout changement portant sur les traits descriptifs entraînerait un changement dans la classe d'objets dénotés et, donc, la constitution d'un nouveau terme.

A partir de la notion de désignateur (substantif) rigide, je propose une hypothèse de travail suivante : un terme peut être employé rigidement dans un discours pour qualifier des objets en les valorisant ou dévalorisant de la même manière qu'un terme peut dénoter rigidement une classe d'objets ${ }^{3}$. Pour conforter la thèse avancée, je ferai appel à la théorie des stéréotypes.

\section{Théorie des stéréotypes}

D’après H. Putnam (1985), (1990), étant radicalement séparée de la fonction référentielle et de l'intension avec laquelle elle ne se confond qu'accidentellement, la signification d'un 
terme n'est pas composée à partir d'un ensemble des primitifs sémantiques, mais de ce qui est dans notre environnement et de la manière dont nous interagissons avec cet environnement. Or, toutes les représentations que nous connaissons ont une association contingente avec leur référent et ne déterminent pas ce que le mot désigne. Il n'y a donc pas de raison de penser qu'une représentation donnée ne sera pas amenée à prendre des significations différentes auprès de groupes différents d'êtres humains ou à changer si la culture ou le monde change. Autrement dit, savoir ce que signifient les mots d'un langage, c'est saisir la manière dont ils sont utilisés par une communauté. Ainsi, H. Putnam propose de définir une forme normale de description de la représentation sémantique attachée à un terme en tant que suite de composants parmi lesquels il distingue les marqueurs sémantiques et syntaxiques, le stéréotype et une description de l'extension. La description sémantique d'eau pourrait avoir donc la représentation suivante :

nom massif, concret (marqueur syntaxique),

substance naturelle, liquide (marqueur sémantique), incolore, transparent, sans goût, apaise la soif, etc. (stéréotype), $H 2 O$ (description d'extension qui capte les propriétés essentielles de l'objet dénoté et permet par là de délimiter l'extension de la classe de ces objets). (Putnam, 1985 : 43).

Le stéréotype dans l'approche de H. Putnam concerne en fait l'usage banal de la langue en tant que pratique de locuteurs individuels : il est formé des traits appartenant aux membres paradigmatiques de la classe en question qui relèvent des idées conventionnelles sur l'apparence, les actions ou la nature d'un $x$. L'information contenue dans le stéréotype n'est pas nécessairement correcte: qu'un trait soit inhérent au stéréotype associé au mot $x$ ne signifie pas qu'il soit vrai et que tous les $x$ le possèdent. 
Cette théorie a été reprise et développée par B. Fradin (1979), (1984) et J.-C. Anscombre (2001). Leur position touche la nature du stéréotype, et peut grosso modo se résumer aux points suivants:

- Le stéréotype n'est pas considéré comme simplement une partie de la représentation sémantique mais avant tout comme un mode d'attribution de la signification. Il s'oppose ainsi à la définition qui consiste à associer analytiquement une signification à un mot à partir de propriétés en nombre fini construites sur la base d'un savoir.

- La représentation sémantique associée à un nom n'est pas la conjonction d'un nombre fini de traits mais une suite ouverte de propriétés qui constituent le stéréotype.

- Cette suite ouverte n'est pas une suite d'éléments mais de phrases de la langue.

En d'autres termes, tout locuteur d'une langue dispose d'une liste plus ou moins longue de termes auxquels il est susceptible d'attacher un certain nombre de caractéristiques sémantiques fixées au sein de la communauté linguistique ${ }^{4}$ à laquelle il appartient au moment où il est locuteur' ${ }^{5}$ L'ensemble de ces caractéristiques stockées sous forme de phrases stéréotypiques - il convient de distinguer ici les phrases en tant qu'entités abstraites, potentialités des énoncés en tant que réalisations de ces potentialités - définit pour un sujet parlant la signification d'un terme considéré. Cet ensemble n'est pas nécessairement le même que celui d'un autre sujet parlant, d'autant plus qu'il peut évoluer ou être modifié pour une raison ou autre. D’où la nécessité de caractériser le stéréotype comme étant une liste ouverte. La façon dont les stéréotypes interviennent dans l'énonciation est à rapprocher de la mise en place d'un thème au sens habituel : le locuteur les convoque, active lors d'une occurrence, même implicitement, comme une idée conventionnellement attachée à un terme, comme une opinion ou un point de vue d'une communauté linguistique à laquelle il appartient. Il ne s'en présente jamais comme l'auteur, mais comme un simple utilisateur. Ce procédé fait donc partie de ce qu'on appelle habituellement les lieux communs. Ainsi, si l'on qualifie l'activité 
de quelqu'un comme travail, on évoque les stéréotypes: Le travail fatigue. Le travail demande des efforts, etc. De même, on explique l'énoncé : Ce tigre est normal / *curieux : il est rayé par la présence dans le stéréotype de tigre de la phrase stéréotypique Les tigres sont rayés qui appartient de manière contingente à la représentation sémantique du nom tigre: si un tigre est albinos et n'est donc pas rayé, il reste néanmoins un tigre.

Voilà brièvement esquissés les fondements du cadre théorique de mon étude. Considérons à présent les caractéristiques essentielles de la représentation sémantique, associée au terme иностранеи, i.e. son stéréotype, relevées dans les discours médiatiques russes.

4. Propriétés de la représentation sémantique attachée au terme иностранец

\subsection{Menace}

Une des propriétés constitutives de la représentation associée à иностранец est définie par la phrase stéréotypique : Иностранщьы представляют для нас опасность. La menace est donc une des caractéristiques dominantes du stéréotype. Cette caractéristique qui reflète le mécanisme de l'individualité sémiotique selon lequel on oppose son espace socioculturel sécurisé à celui d'autrui considéré comme dangeureux (Лотман, 1996 : 175) se réalise par exemple dans les énoncés (1) où le terme s'emploie en tant que sujet grammatical faisant ainsi le thème de l'énonciation ou en tant que objet de l'action. Les verbes co-occurrents du terme souvent dotés des modalités dépréciatives renforcent la propriété du stéréotype.

(1) Иностраниьь съедают наши банки. [Аргументы и факты, 29.01.2003]

Если иностранцы скупят нашу финансовую систему, денежные потоки из страны уйдут. [Вслух о., 24.10.2003]

Если энергетику и газовое хозяйство будут контролировать иностранцы, не склонные к благотворительности, цень на тепло, свет и другие коммунальнье услуги окажутся для большинства из нас просто неподъемными. [Аргументы и факты, 29.03.2005] 
А в наши дни отдали на откуп иностранщам почти все Берингово море. (...) Может, тогда бы и рыба досталась нам, а не иностранцам, и была бы у рыбаков работа. [Рьббак Приморья, 23.01.2003]

Вторая возможная угроза - уход сырьевых запасов под контроль иностранщев. [Аргументы и факты, 08.04.2002]

Богатства наџчи оказываются за гроши у иностранцев [Новая газета, 09.01.2003]

La caractéristique de menace constituant le stéréotype est également explicitée à travers les locutions appartenant aux contextes historiques ancrés dans la mémoire collective russe et celles qui relèvent du passé récent. Ainsi, la séquence массовое нашествие иностранцев dans (2) renvoie à l'expression phraséologique bien connue мамаево нашествие qui évoque le fléau du joug mongol, période douleureuse de l'histoire russe, et convoque l'image de «l'incarnation du mal» dans la Russie médievale. De même, les constructions болтать иностраниу помогать [www.utro.ru] et иностранцы не дремлют [Известия, 09.08.2004] font référence aux slogans : «Болтать - врагу помогать» и «Враг не дремлет» devenus expressions courantes et associent ainsi les étrangers aux ennemis issus des affiches de propagande des années 1950-1960. ${ }^{6}$

(2) Итак, само по себе разрешение учреждать филиаль зарубежных банков в обозримой перспективе вряд ли привело бы к массовому намествию иностранцев и мало изменило бы соотношение сил между основными участниками рынка финансовых услуг. [Эксперт, 06.12.2004]

\subsection{Peur/Méfiance}

L'idée de peur et de méfiance des russes vis-à-vis des étrangers est directement liée à la caractéristique de menace. Elle est manifeste à travers la phrase stéréotypique Иностранцам доверять нельзя actualisée dans les énoncés (3). 
(3) Опасность присутствия иностранщев на рынке. [Независимая газета, 28.04.2003]

Мы боимся пустить иностранцев на рынок. [Российская газета, 15.11.2002]

Российские банкиры опасаются конкурениии со стороны иностранцев. [КоммерсантъDaily, 20.01.2005]

Les locutions à dimension socioculturelle : proverbes, dictons, idiotismes, etc., jouent un rôle important dans le processus de stéréotypisation. Adaptées au besoin du contexte par toute sorte de remaniement: remplacement, ajout ou insertion d'un ou plusieurs éléments, troncation, suppression d'une marque syntaxique, etc., ces expressions ne sont pas employées en tant que des simples références socioculturelles mais comme des éléments du discours qui ne produisent pas le sens mais fournissent des repères par rapport auxquels le discours s'organise et prend forme. Elles sont perçues dans leur intégralité même si leur forme est en partie modifiée. Mentionnons à titre d'exemple la séquence иностранцев бояться - в лес не xодumb. [www.lenta.ru] créée par association au proverbe russe connu où le mot иностранцы de par le cadre proverbial où il est employé, fait écho au nom волки dont le stéréotype renvoie à l'image d'un animal dangereux.

La représentation sémantique de иностранец reflète également à travers les phrases stéréotypiques Иностранцыь нам не доверяют. Иностранцы нас боятся la peur et la méfiance des étrangers envers la Russie et les russes. Cette caractéristique est illustrée par les énoncés (4) :

(4) Иностранцы перестраховываются. [Известия, 05.03.2002]

Иностранцьь чуть-чуть в нас поверили. Боятся, но все-таки к нам едут [Известия, 14.07.2002]

Тревоги иностранцев хорошо известны. [Известия, 19.09.2002]

Кредитовать строчтельство в России иностранць боятся, хорошо зная порядки, цุарящие на наших заводах. [Известия, 31.07.2001] 


\subsection{Protection/Défense}

Le sentiment de menace, de peur et de méfiance implique l'opposition, la défense, la protection de son espace socioculturel. Or cette propriété, définie par la phrase Иностранцам надо противостоять, fait partie intégrante du stéréotype de l'étranger. On trouve son actualisation dans les énoncés (5), ainsi que dans certaines expressions phraséologiques, comme par exemple, шиш этим иностранцам с маслом [www.strana.ru], задать иностранияам периу [www.sovsekretno.ru], никогда нам не быть с иностраниами в одной упряжке [Политком.ру, 12.08.2003], etc., utilisées en tant que parole d'une autorité anonyme pour appuyer ce que l'on avance. Le rôle principal de ce genre de constructions consiste à rattacher des éléments diégétiques au discours et renforcer ainsi la vraisemblance des faits narrés.

(5) Запретить иностранцам лезть в нашу историю, нашу культуру. [Известия, 18.07.2002]

Продавать надо не иностранцам, а своим [Газета, 09.01.2003]

Офищиально российские авиаторы просили 10 лет защиты от иностранщев, но в частных беседах соглашались на те же 6. [Известия, 14.03.2002]

Средства компании направлены на борьбу с несносными иностранщами [Рекламный мир, 31.03.2003]

Надо потеснить иностранщев. [Известия, 24.07.2003]

\subsection{Mépris/Ironie}

L'attitude de mépris et d'ironie vis-à-vis des étrangers est une propriété intégrante de la représentation associée au nom иностранец dans les médias russes. Cette propriété est fixée à travers la phrase Иностранцы ничего у нас не понимают constituant le stéréotype du terme et actualisée, entre autres, dans les constructions adjectivales à connotation ironique ou péjoratives du type : важные, несносные, хитрые, неискушенные, наивные, глупые, 
приличные, дорогие... иностранщы аinsi que dans des expressions phraséologiques axées sur la dénonciation ironiques des étrangers comme, par exemple, иностранец - человек высшей касть [Известия, 24.01.2003], иностранщьь - белая кость [Известия, 11.17.2002], иностранеи фигов [Столища, 24.11.2005], иностранеи, ежкин кот! [Бизнес-журнал, 03.03.2004], etc. Ayant comme fonction de renforcer la connivence avec le lecteur en mobilisant l'univers d'un discours familier, ces séquences sont censées faire partager de façon inconsciente les idées stéréotypées que le locuteur transmet. La caractéristique de mépris et d'ironie est explicitée dans les énoncés (6).

(6) А иностранщев дорогих теперь учат еще одному слову, кроме стандартных "зджавствуйте", "спасыибо" и "ери твою медь". [Известия, 21.04.2002]

Известия нашли следы отдельных наивных иностранщев, прослышавщих, что в России, напряженно работающей над улучшением своего международного имиджа под водительством лучших "пиар-умов" странь, что-то такое стало меняться к лучшему. [Известия, 23.04.2002]

Одно слово: он - иностранец со всеми вытекающими отсюда последствиями. [Солдат удачи, 11.03.2004]

\subsection{Utilité}

Malgré la dominance des propriétés nettement négatives associées à иностранеu, il se trouve tout de même que certaines caractéristiques attribuées aux étrangers dans les médias russes sont orientées positivement. Une de ces propriétés constituant le stéréotype est : Иностранциь нам нужныл. Иностранщь приносят пользу. Cette propriété est reflétée notamment dans les énoncés (7).

(7) У иностранщев есть чему поучиться. [Мир \& Дом. Сity, 15.06.2003]

Нововведением мы обязаны иностранщам. [Столища, 06.01.2003] 
Иностранцы приезжают, вкладьвают деньги и нормально организуют работу на земле. [Дело, 17.06.2002]

России как воздух нужны иностранщьы. [Известия, 20.02.2002]

Иностранцы способствуют исчезновению у россиян комплекса провинциальности. [Коммерсанть-Daily, 26.01.2003]

Иностранцыь обучают персонал, стремятся, чтобы не уходили, не болели, повышали профессиональный уровень. [Знание - сила, 2003]

\section{Conclusion}

Cette breve analyse du stéréotype associé au nom иностранеи me conduit à poser deux thèses en guise de conclusion.

1. Le contenu lexical du terme est constitué de deux ensembles de caractéristiques sémantiques discrimés par l'interprétation référentielle/non référentielle que reçoit le substantif dans le discours. D'une part, иностранеи est employé et interprété comme référent à une entité extra-linguistique. Dans ce cas, il s'agit des propriétés classifiantes, i.e. constitutives d'un objet de discours, auxquelles sont attachées des phrases définitionnelles telles que : Иностраниь имеют чужое гражданство. Иностранцыь живут заграницей., etc. D'autre part, le terme est employé et interprété dans des constructions dites qualificatives où il qualifie l'objet en le dévalorisant ou valorisant. Il s'agit alors des propriétés non classifiantes, i.e. constitutives d'une qualification, qui sont culturellement associées au terme sous forme de phrases stéréotypiques formant le stéréotype du nom ${ }^{7}$.

2. Les propriétés de la représentation sémantique de иностранеи relevées dans les discours médiatiques russes laissent supposer que son stéréotype possède deux profils distincts :

- le profil dominant иностранец - враг constitué des phrases stéréotypiques :

Иностранцы представляют опасность.

Иностранцุам нельзя доверять.

Иностранщьы ничего не понимают. 
Иностранцам надо противостоять.

- et le profil plutôt périphérique иностранеu - партнер constitué des phrases du type : Иностранщы нам нужны.

Иностранцы приносят пользу.

La dominance du profil négatif qui ressort à une certaine manière d'appréhender la réalité est déterminée par le phénomène d'ethnocentrisme, cette vocation de toute société à mesurer les différences à l'aune de sa propre culture. L'ethnocentrisme correspond donc à une attitude générale des groupes sociaux à l'égard de ce qui diffère d'eux par des caractères ethniques, culturels ou nationaux et consiste à rejeter tout ce qui n'est pas conforme à la norme sous laquelle on vit. Il apparaît alors la chose du monde la mieux partagée et, de ce point de vue, la communauté russe ne se distingue pas des autres. ${ }^{8}$

\section{REFERENCES BIBLIOGRAPHIQUES}

1. Anscombre, J.-C., « Le rôle du lexique dans la théorie des stéréotypes », Langages, $\mathrm{N}^{\circ}$ 142, 2001, pp. 57-76

2. Fradin, B., «Anaphorisation et stéréotypes nominaux », Lingua, 64, N4, 1984, pp. $325-369$

3. Fradin, B., Marandin J.-M., «Autour de la définition: de la lexicographie à la sémantique », Langue Française, №43, 1979, pp. 60 - 83

4. Putnam, H., «Signification, références et stéréotype », Philosophie, №5, 1985, pp. $21-44$

5. Putnam, H., Représentation et réalité, Paris, Gallimard, 1990

6. Roze, X, Stéréotypes sociaux, Encyclopaedia Universalis, Paris, 2002, pp. 614-615 
7. Ефремова, Т. Ф., Новый словарь русского языка. Москва, Русский язык, 2000

8. Лотман Ю.М., Внутри мыслящих миров. Человек - текст - семиосфера история., Москва, ЯРК, 1996

9. Ожегов, С. И., Словарь русского языка, Москва, Русский язык,1981

10. Ушаков, Д. Н., Словарь русского языка, Москва, Астрель, АСТ, 2000

\section{NOTES}

${ }^{1}$ Le terme 'représentation sémantique' utilisé ici par commodité n'a pas la même valeur que celle que lui donnent la plupart des théories sémantiques. Ce terme sera interprété dans le présent article de la même manière que dans Fradin, 1984, i.e. il renverra au stéréotype composé des phrases associées à $x$ de façon stable dans un contexte socio-culturel donné pour un ensemble de personnes donné.

${ }^{2}$ «Национальный корпус русского языка»: http://www.ruscorpora.ru

${ }^{3}$ Je m'appuie ici sur l'hypothèse de B. Fradin et J.-M. Marandin (1979)

${ }^{4}$ On entend sous la communauté linguistique « un ensemble de sujets parlants partageant une certaine liste de termes affectés des mêmes significations ». (Anscombre, $2001: 60$ )

${ }^{5}$ Un même sujet parlant peut appartenir à diverses communautés à des moments différents où il est locuteur. Par conséquent, certaines phrases à l'intérieur du stéréotype peuvent être antinomiques, erronées, voire carrément fausses. Ce qui montre d'ailleurs que le stéréotype ne décrit pas la réalité. Pour plus de précisions, je renvoie à Anscombre, 2001.

${ }^{6}$ Les auteurs de ces affiches créées respectivement en 1954 et 1961 sont В. Б Корецкий, О.

М. Савостюк еt Б. А.Успенский

${ }^{7}$ Voir à ce sujet Fradin, Marandin, 1979

${ }^{8}$ Je renvoie à Лотман,1996 et Roze, 2002 
